\title{
ASPECTOS FITOQUÍMICOS Y ACTIVIDAD CICATRIZANTE DE Senecio culcitoides Weed
}

\author{
MARCELA Y. SORIANO S.' , PABlo E. BONILla R.' , JORGE L. ARROYO A. Y SONIA PEREYRA
}

\section{RESUMEN}

La especie vegetal en estudio fué ubicada y recolectada en el mes de Febrero en la localidad de Ticlio situada entre 4500 a 5000 m.s.n.m. a la altura del km 130 de la carretera central, en la zona de sierra del departamento de Lima. Fué clasificada en el Museo de Historia Natural como: Senecio culcitoides Weed. de nombre vulgar: Huira-Huira, Huajchor, Semaro-Huamasti (1), para la extracción de los metabolitos secundarios se realizó maceración etanólica de hojas de Senecio culcitoides Weed continuándose con ensayos de solubilidad y el screening fitoquímico respectivo, encontrándose mayor canticlad de compuestos fenolicos, como flavonoides, taninos, etc., además de alcaloides, esteroides y/o triterpenoides. La elucidación estructural mediante espectroscopía ultravioleta - visible, determinó que pertenecían a compuestos fenólicos tipo flavonoides derivados del núcleo flavanonona (5,7yihidroxi-4',6,8-trimetoxiflavanonona; $3^{\prime}, 5$-dihidroxi-4'-metoxi-7-O-rhannoglucosyl flavanonona) y una chalcona (3,4dihidroxichalcona). La evaluación de la actividad cicatrizante del extracto al $20 \%$ en forma de crema fue de acuerdo al método tensiométrico, estadísticamente, se observó que los niveles de resistencia a la tensión del extracto etanólico del Senecio culcitoides Weed presentó una diferencia significativa de $\mathrm{P}<0.05$ al compararse con el control que es la crema base cetiol lanette $6 \%$ y como standard el fármaco "vibe» o sangre de grado al $1 \%$, resultados que fueron corroborados con los respectivos cortes histológicos.

Palabras clave: Senecio culcitoides Weed, compuestos fenólicos, flavonoides, crema del extracto al $20 \%$, crema base cetiol lanette $6 \%$, Sangre de grado $1 \%$, actividad cicatrizante.

\section{SUMMARY}

The vegetable species in study were located and gathered in the month of February in the town of Ticlio located between 4500 to 5000 o.s.l. to the height of the $\mathrm{km} 130$ of the central highway, in the area of mountain of the department of Lima. It was classified in the Museum of Natural History as: Senecio culcitoides Weed. of vulgar name:, Huajchor, SemaroHuamasti, According to Jaroroslav Soukup (1). For the extraction of the secondary metabolites was realized a maceration etanólica of leaves of Senecio culcitoides Weed being continued solubility test and the screening respective fitoquimico, being bigger quantity of phenolic compound, as flavonoides, tannins, elc., besides alkaloids, steroids and/or triterpenoides. The structural elucidation by means of ultraviolet espectroscopía - visible, was determined that they belonged to phenolic compound derived flavonoides belong the nucleus: flavanonona $\left(5,7\right.$ dihidroxi-4',6,8-trimetoxiflavanonona; $3^{\prime}, 5$-dihidroxi4'-metoxi-7-or-rhannoglucosyl flavanonona) and a chalcona (3,4-dihidroxichalcona).

The evaluation of the healing activity of the extract at $20 \%$ in form of cream was according to the tensiométrico method, statistically, it was observed that the resistance levels to the tension of the etanólic extract of the Senecio culcitoides Weed presented a significant difference of $\mathrm{P}<0.05$ being compared with the control that it was a cream base cetiol lanette $6 \%$ and with the standard one the fármaco Vibe (sangre de grado $1 \%$ ), those results were corroborated with the respective histology.

Key words: Senecio culcitoides Weed, phenolic compound, flavonoides, cream of the extract to $20 \%$, basic cream cetiol lanette $6 \%$, Sangre de yrado $1 \%$, healing activity.

\section{INTRODUCCIÓN}

El género Senecio perteneciente a la Familia Asteraceae, consta de unas 1500 especies cosmopolitas, en el Perú existe la mayor diversidad con 180 especies que habitan en regiones de altitudes superiores a los 4500 m.s.n.m., $(2,3)$ además de contener entre sus metabolitos alcaloides pirrolizidínicos presentan otros, en su mayoría

'Instituto de Investigación en Ciencias farmacéuticas y Recursos Naturales «Juan de Dios Guevara» Facultad de Farmacia y Bioquímica de la Universidad Nacional Mayor de San Marcos. flavonoides, taninos, terpenos, sesquiterpenlaclonas, etc., los cuales son una fuente potencial de sustancias biológicamente activas de aplicación terapéutica.

El presente trabajo tiene como objetivo elucidar la estructura química de algunos metabolitos secundarios presentes en las hojas del Senecio culcitoides Weed. y evaluar la actividad cicatrizante y proponer una alternativa de empleo farmacológico de esta especie vegetal. 
MATERIAL Y MÉTODOS

\section{Material vegetal}

La especie vegetal se recolectó en el mes de febrero en la localidad de Ticlio (zona de sierra de Lima, entre el $\mathrm{Km} 125$ al 130 de la carretera central) entre los 4500 a 5000 m.s.n.m.

Preparación del extracto. Se maceraron $300 \mathrm{~g}$ de polvo de las hojas en $800 \mathrm{ml}$ de etanol al $95 \%$ durante siete días, posteriormente el líquido filtrado se concentro a temperatura inferior a $40^{\circ} \mathrm{C}$. hasta obtener el extracto seco etanólico.

Marcha de solubilidad. En solventes de polaridad creciente.

Screening fitoquímico. La detección de los constituyentes químicos de los extractos se realizó siguiendo la marcha fitoquímica general (4)

Ensayos cromatográficos:

Cromatografía analítica preliminar. El extracto etanólico se desarrolló en cromatoplacas con el sistema de solventes: cloroformo: metanol 9:1 y butanol: ácido acético: agua 4:1:5 (5); que se ha revelado con tricloruro férrico, $\mathrm{H} 2 \mathrm{SO} 450 \%$ y vapores de amoniaco, observándose a la luz visible y bajo la lámpara de luz UV de 254 y $366 \mathrm{~nm}$, detectando la presencia de flavonoides y otros metabolitos secundarios.

Cromatografía en capa fina a escala preparativa. Se realizó la siembra en banda en cromatoplacas de Silicagel G-60 de $20 \times 20 \mathrm{~cm}$ para obtener las fracciones respectivas.

Evaluación de la actividad cicatrizante

Se realizó siguiendo el método tensiométrico Modelo de Vaisberg et al. (6) ulilizándose ratas albinas Sprague dawley con un peso aproximado de 200 a $220 \mathrm{~g}$. Fueron preparadas cremas de los extractos al $20 \%$ comparándose con el control (crema base cetiol lanette $6 \%$ ) y el fármaco Vibe (Sangre de grado al 1\%), administrados por vía tópica durante siete días, el último día de tratamiento los animales fucron sacrificados y mediante un dinamómetro adaptado se buscó abrir las lesiones cicatrizadas, expresándose la fuerza de tensión en gramos.

\section{RESULTADOS}

\section{ESTUDIO QUÍMICO}

Tabla $\mathbf{N}^{\circ}$ 1. Marcha de solubilidad del extracto etanólico de hojas de Senecio culcitoides Weed

\begin{tabular}{|l|c|}
\hline SOLVENTES & GRADO DE SOLUBILIDAD \\
\hline n-hexano & - \\
cloroformo & + \\
acetato de etilo & + \\
butanol & + \\
etanol & +++ \\
metanol & ++ \\
agua & - \\
\hline
\end{tabular}

Tabla $\mathbf{N}^{\circ} 2$. Marcha fitoquímica del extracto etanólico de hojas de Senecio culcitoides Weed

\begin{tabular}{|l|c|}
\hline METABOLITOS & CONCENTRACIÓN \\
\hline Carbonilo & ++ \\
Glicósidos & ++ \\
Compuestos fenólicos & +++ \\
Taninos & ++ \\
Flavonoides & +++ \\
Alcaloides & ++ \\
Esteroides y / o terpenoides & ++ \\
Lactonas & ++ \\
\hline
\end{tabular}

Tabla $\mathrm{N}^{\circ} 3$ Análisis cualitativo de las fracciones del extracto etanólico por ccf.

\begin{tabular}{|c|c|c|c|c|}
\hline Frac. $R f$ & Uv $366 \mathrm{~nm}$ & Vapóres $\mathrm{NH}_{4}+\mathrm{Uv}$ & $\mathrm{FeCl}_{3}$ & $\mathrm{H}_{2} \mathrm{SO}_{4} 50 \%$ \\
\hline $1 \quad 0.35$ & Púpura intenso & Celeste & Mancha negrusca & Verde limón \\
\hline $2 \quad 0.54$ & Púrpura & Celeste & Mancha negrusca & Verde limón \\
\hline $\begin{array}{ll}3 & 0.84\end{array}$ & Amarillo & Naranja & Mancha negrusca & Verde limón \\
\hline
\end{tabular}




\section{ESTRUCTURAS QUIMICAS PROPUESTAS}

Compuesto (1) $1284,329 \mathrm{~nm}$.

Compuesto (2) $1283,326 \mathrm{~nm}$.

Compuesto (3) I 265, 316, $365 \mathrm{~nm}$. Por comparación con estructuras propuestas por Mabry (5) y reacciones químicas.<smiles>[R][R]c1c([R])c([R])c2c(c1[R])OC(c1ccc([R])c([R])c1)CC2=O</smiles>

Compuesto (1) (5,7-DIIIIDROXI-4', 6, 8 TRIMETOXIFLAVANONONA) $4^{\prime}, 6,8=\mathrm{R}=\mathrm{OCH} 3$ $; 5,7=\mathbf{R}^{\prime}=\mathrm{OH}$

Compuesto (2) (3',5 -DIHIDROXI-4'-METOXI7-O-RHAMNOGLUCOSILFLAVA

NONONA) $4^{\prime}=\mathrm{R}=\mathrm{OCH} 3 ; 3^{\prime}, 5=\mathrm{R}^{\prime}=\mathrm{OH} ; 7=$ $\mathbf{R}^{\prime \prime}=$ O-RHRAMNOGLUCOSIL<smiles>O=C(/C=C/c1ccc(O)c(O)c1)c1ccccc1</smiles>

Compuesto (3) (3,4-DIHIDROXICHALCONA)
ESTUDIO FARMACOLÓGICO:

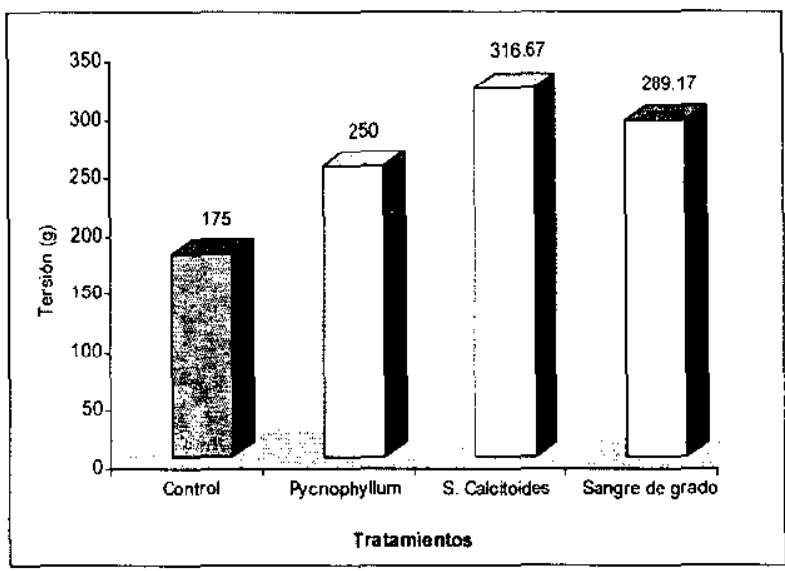

Figura $\mathrm{N}^{\circ} 1$. Actividad cicatrizante de la crema a base del extracto etanólico de Senecio culcitoides Weed (expresado en promedio de la fuerza de tensión en gramos para abrir la herida cicatrizada)

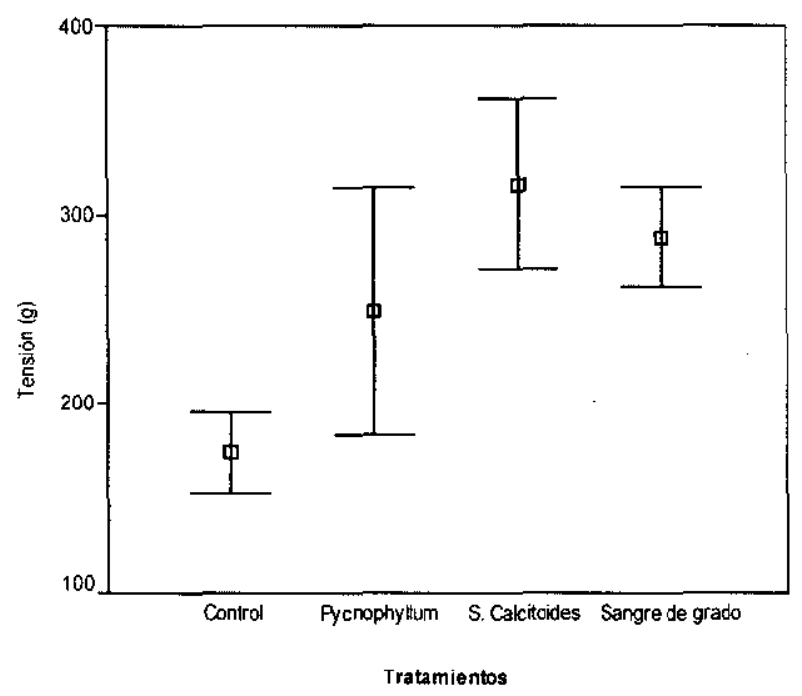

Figura $\mathrm{N}^{\circ \circ}$. Análisis de varianza de la actividad cicatrizante de Senecio culcitoides Weed.

\section{ANOVA}

\begin{tabular}{|c|c|c|c|c|c|}
\hline & Suma de cuadrados & gl & Media cuadrática & S & Sig. \\
\hline TRATAMIENTO Inter-grupos & $68,194.792$ & 3 & $22,731.597$ & 13.331 & .000 \\
Intra-grupos & $34,104.167$ & 20 & $1,705.208$ & & \\
Total & $102,298.96$ & 23 & & & \\
\hline
\end{tabular}




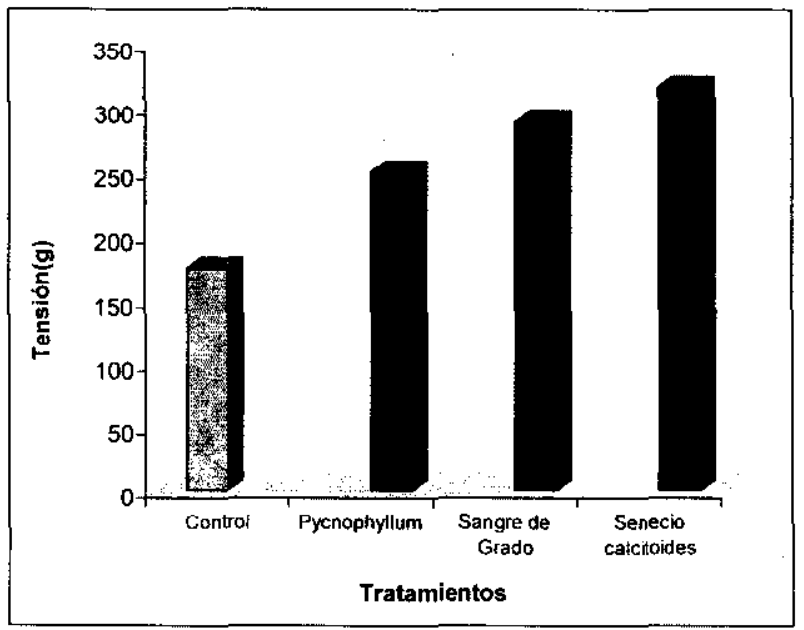

Figura $\mathrm{N}^{\circ} 3$. Estadística comparativa según Prueba de Tukey de la fuerza de tensión expresada en gramos para abrir la herida cicatrizada por Senecio culcitoides Weed.

\section{DIscusión}

Se puede observar en la Tabla $\mathrm{N}^{\circ} 1$, mayor solubilidad en los solventes polares que indicaría, presencia mayoritaria de compuestos fenólicos de alta polaridad. Las determinaciones cualitativas en el extracto etanólico mostrados en la Tabla $N^{\circ} 2$, revelan la presencia de compuestos fenólicos, taninos, flavonoides, sesquiterpenlactonas, esteroides $\mathrm{y} / \mathrm{o}$ triterpenos, destacando como constituyentes mayoritarios los flavonoides.

Como se observa en la Tabla $N^{\circ} 3$, se obtuvieron tres fracciones, las cuales de acuerdo a las reacciones de reconocimiento, que luego fueron leídas en el espectrofotómetro ultravioleta para su elucidación estructural, determinándose de esta manera que pertenecían a compuestos fenólicos tipo flavonoides siendo derivadas del núcleo flavanonona $(5,7-$ dihidroxi-4', 6, 8 -trimetoxiflavanonona; 3', 5dihidroxi-4'-metoxi-7-O-rhannoglucosylflavanonona) y una chalcona (3,4-dihidroxichalcona).

De los ensayos para evaluar la actividad cicatrizante, se observó que la crema al $20 \%$ del extracto, produjo mejor resultado comparado con el estándar fármaco "vibe» o sangre de grado al $1 \%$ y el control (cetiol lanctte $6 \%$ ), debido a que presento una mayor fuerza de tensión además que reflejo una mayor velocidad de reparación celular en los primeros días de cicatrización, en la fuerza de tensión aplicada para cada grupo se pudo determinar el promedio, el análisis de varianza y la prueba de Tukey, en los tres se puede comprobar el grado de efectividad que presenta la crema del extracto al $20 \%$ comparándose con el estándar (sangre de grado al 1\%), el control (cetiol lanette 6\%) e incluso con otro extracto como es el de Picnofillyum glomeratum. Figuras $\mathrm{N}^{\circ} 1,2,3$.

Se sabe que los senecios tienen buena actividad en el tratamiento del asma bronquial (7), y también se han reportado resultados óptimos en el efecto antiinflamatorio, analgésico, antioxidante (8) y ahora se reporta el efecto cicatrizante. Estos datos fueron corroborados con la histología donde se observa que la crema del extracto al $20 \%$ produjo mayor cantidad de tejido de granulación, neovasos, fibroblastos, y presencia de tejido colágeno central, comparados con el estándar, donde se observa escaso tejido de granulación, tapón superficial y neovasos, el control presenta escasos neutrofilos, fibrina e inclusive hemorragia. En la cicatrización interfiere la inflamación y el daño celular. Es sabido que los flavonoides evitan la liberación de prostaglandinas, histaminas, evitan la migración de elementos formes (neutrófilos y otros) que están relacionados con la cascada de la inflamación, además estabilizan la membrana celular capturando a los radicales libres. presentes evitando daño celular y activando el sistema de complejo bioquímico para la regeneración del tejido (9).

El extracto extraído de hojas de Senecio culcitoides Weed en forma tópica presenta una muy buena. actividad cicatrizante.

\section{REFERENCIAS BIBLIOGRÁFICAS}

1. Soukup J. 1987. Vocabulario de los nombre vulgares de la Flora Peruana y Catálogo de los Géneros. Editorial Salesiana. Lima.

2. Brako L, Zaruchi J. 1993. Catalogue of flowering Plants and Gymnosperm of Perú. Monograph in Sistematic Botany from the Missouri Botanical Garden.

3. Classification and diversity of Asteracea in Peru. Certificado (Base de datos en Internet) (Actualizado sept. 2003) hltp://www.sacha.org/ fenil/aster/peruaster.hrml

4. Lock O. 1994. Investigación Fitoquímica. Métodos de estudios de productos naturales. Fondo Editorial PUC P. $2^{\circ}$ Edición, Lima.

5. Mabry T. Markham K. and Thomas M. 1970. The systematic identification of flavonoids (New York) Springer Verlag. 
6. Villegas L, Fernández I, Maldonado $\mathrm{H}$, Torres $\mathrm{R}$, Zavaleta A, Vaisberg A, et al. 1997. Evaluation of the wound healing activity of selected traditional medicinal plants from Perú. J of Ethnopharmacology 55(3): $193-200$.

7. Bragg A. 1999. Diccionario Enciclopédico de Plantas útiles del Perú. Centro de estudios regionales andinos Bartolomé de las Casas. Programa de las Naciones Unidas para el Desarrollo.
8. Bonilla, P.; Lozano, M.; Arroyo ,J. et al. 2003. Acción antiinflamatoria, antioxidante y analgésica de compuestos fenólicos de Senecio serratifolius. VI jornadas de investigación en Ciencias Farmacéuticas y Bioquímicas «Dr. Fernando Montesinos Ampuero». Lima, 06- 08 de agosto.

9. Havsteen. B.2002. The biochemistry and medical significance of the flavonoids. Pharmacology \& Therapeutics 96: 135- 202. 